\title{
THE POSSIBILITY OF NONINVASIVE MICRON HIGH ENERGY ELECTRON BEAM SIZE MEASUREMENT USING DIFFRACTION RADIATION
}

\author{
G.Naumenko\#, A.Potylitsyn, Tomsk Polytechnic University, Tomsk,Russia \\ S.Araki, A.Aryshev, H.Hayano, P.Karataev, T.Muto, J.Urakawa, KEK, Tsukuba,Japan \\ M.Ross, Stanford Liner Accelerator Center \\ D.Cline, Y.Fukui, University of California at Los Angeles, Department of Physics and Astronomy \\ R.Hamatsu, Tokyo Metropolitan University, Tokyo,Japan
}

\begin{abstract}
A new method based on diffraction radiation emitted by charged particles moving through a slit between two flat rectangular plates inclined with respect to each other around the axis perpendicular to the slit has been suggested. The theoretical model for calculating the ODR radiation from such targets (including focusing by cylindrical lens) is presented. It is shown that the sensitivity of this method does not depend on the Lorenzfactor directly. The target with the "dis-phased" angle 6.2 milliradians and the slit width 425 microns was manufactured for experimental test. Some preliminary experimental results are presented.
\end{abstract}

\section{INTRODUCTION}

Recently the project for development of the noninvasive diagnostics method using optical diffraction radiation (ODR) of electrons, passing through a flat conductive slit based on the work [3], has been in progress. This technique has been successfully applied for beam size measurements at KEK: Accelerator Test Facility (ATF) $[1,2]$. It has been shown that the beam size as small as $14 \mu \mathrm{m}$ can be measured through the analysis of the ODR angular distribution.

However for the high electron energy a relative contribution of a synchrotron radiation background, which can not be separated from the ODR, limited the method sensitivity to the beam size catastrophically.

\section{ODR METHOD MODIFICATION}

We have developed a new beam size measurement technique using ODR characteristics from an electron passing through a slit between two flat plates inclined with respect to each other at an angle $2 \alpha$ (see Fig.1).

A lens installed in the light pass leads two cones in the detector plane producing an interference pattern, which is completely different from the ODR distribution from a plane slit. The visibility of the interference fringe significantly depends on the transversal beam size.

Moreover, the sensitivity in this case is determined by the angle $\alpha$ and the DR observation wavelength and does not depend on the Lorentz-factor $\gamma$. This fact allows us to use this method for large energy electron beams.

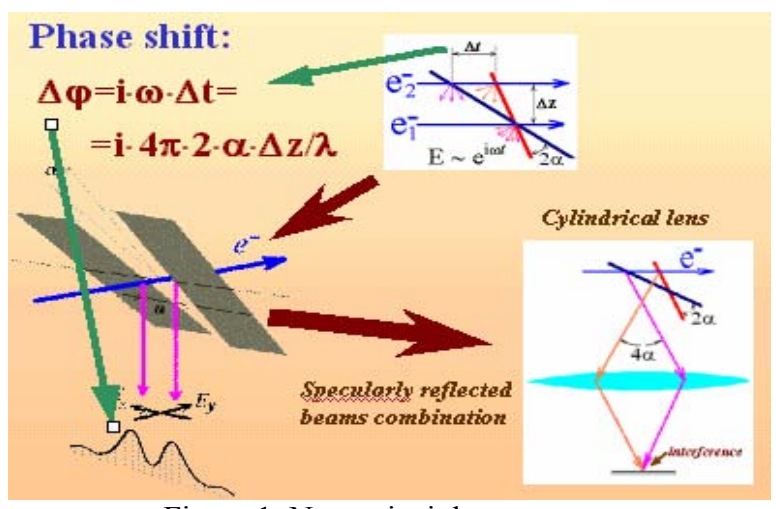

Figure 1: New principle geometry.

\section{Principles of the Theoretical Approach}

The theoretical analysis of the radiation process and radiation evolution was built beginning from the Fourier transform of the incident charged particle field. To obtain an electromagnetic field in the optical lens plane we integrate the incident particle field over the target surface

And finally we take into account a field transformation by cylindrical optical lens and integrate the electromagnetic field over the lens surface.

In approximation of infinite vertical target size and infinite lens size this procedure may be simplified to a simple expression, which allows us to calculate the radiation intensity distribution in the detector plane (Fig.2) for experimental condition described below.

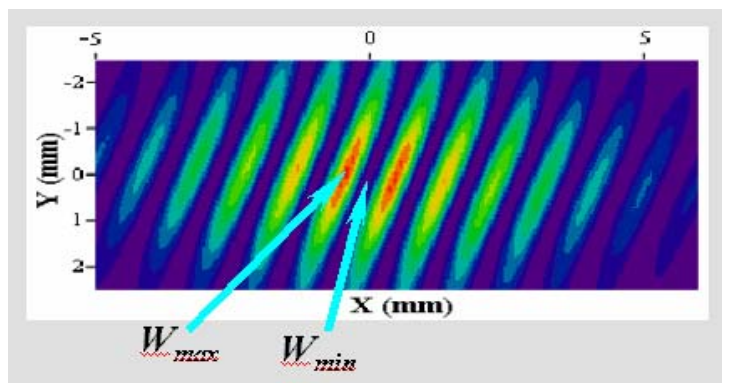

Figure 2: Theoretical radiation intensity image in detector plane for experimental conditions. 
The difference between maximal intensity $\boldsymbol{W}_{\max }$ and minimal intensity $\boldsymbol{W}_{\boldsymbol{m} \text { in }}$ may be used for calculating the vertical beam size. We define a visibility as

$$
4 \pi \frac{\sigma \alpha}{\lambda}=\sqrt{\frac{\ln \left(V^{-1}\right)}{2}}
$$

Then the vertical beam size $\sigma$ may be found from the following dependence.

$$
V=\frac{W_{\max }-W_{\min }}{W_{\max }+W_{\min }}
$$

So if we measure a radiation intensity distribution in the detector plane like Fig.7, we can calculate a vertical beam size. It is important, that this beam diagnostics method is non-invasive and the method sensitivity does not slope down for extremely high electron energies.

\section{EXPERIMENTAL TEST OF ODR FROM A NEW TARGET}

The experiment was performed at the extraction line of Accelerator Test Facility KEK ATF in Japan. On Fig.7 is shown the experimental setup, used for this experiment.

Table 1. Target parameters

\begin{tabular}{c|c|c|c}
\hline Target № & $\boldsymbol{\beta}$ (mrad) & $\boldsymbol{\alpha}$ (mrad) & Slit width $(\boldsymbol{\mu m})$ \\
\hline 5 & $0.75 \pm 0.1$ & $6.2 \pm 0.1$ & $425 \pm 3$ \\
\hline
\end{tabular}

The experiment was performed at next experimental conditions, including beam parameters (Table 2), physical conditions (Tab. 3) and target parameters (Tab.1, Fig.3).

Table 2. Electron beam parameters

\begin{tabular}{|l|l|}
\hline Electron energy & $1.28 \mathrm{GeV}$ \\
\hline Bunch population & $1.2 \times 10^{10}$ \\
\hline Horizontal emittance & $1.4 \times 10^{-9} \mathrm{~m} \cdot \mathrm{rad}$ \\
\hline Vertical emittance & $1.5 \times 10^{-11} \mathrm{~m} \cdot \mathrm{rad}$ \\
\hline
\end{tabular}

Table 3. Physical conditions

\begin{tabular}{|l|l|}
\hline Lorenz-factor $(\boldsymbol{\gamma})$ & 2505 \\
\hline Wavelength $(\boldsymbol{\lambda})$ & $558 \mathrm{~nm}$ \\
\hline Distance from target to detector $(\mathbf{L})$ & $3.56 \mathrm{~m}$ \\
\hline $\mathbf{L} / \gamma^{2} \lambda$ & 1.1 \\
\hline Focus length & $150 \mathrm{~mm}$ \\
\hline
\end{tabular}

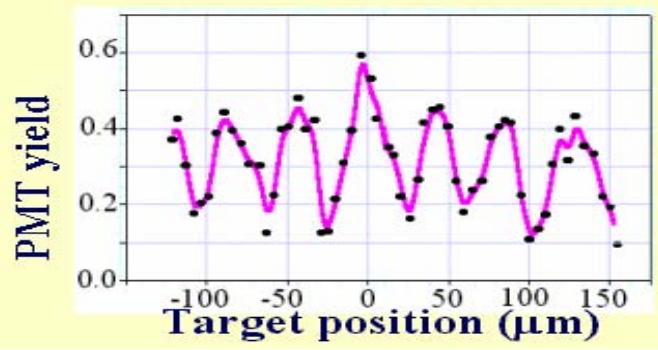

Figure 4: Radiation intensity image in detector plane measured using photo-multiplier.

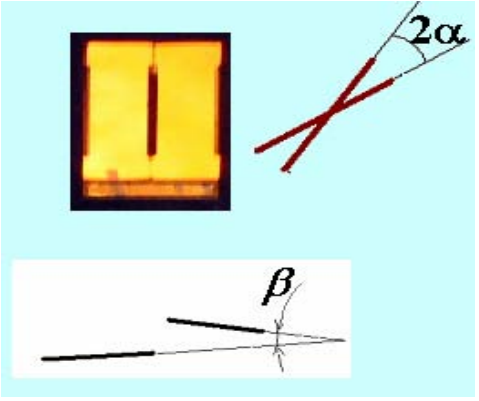

Figure 3. Target parameters.

\section{Preliminary Experimental Results}

The measured radiation intensity distribution in the detector plane similar to the theoretical one (Fig.2) is shown in Fig.4. The measurements were done using photo-multiplier. $0.25 \times 0.64 \mathrm{~mm}$ collimator was used in front of the photo-multiplier. The vertical and horizontal scan was performed by rotation of the reflected mirrors.

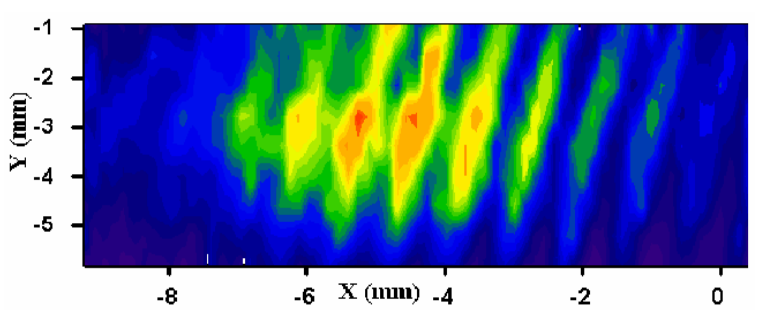

Figure 5: Measured dependence of the radiation yield as a function of the vertical target position.

We can see a good agreement with the theoretical dependence shown in Fig.2, but a bad spatial resolution was obtained because of using the photo-multiplier with fixed collimation. The dependence of the radiation yield as a function of the vertical target position was measured (Fig.5) using the same collimator.

A similar theoretical dependence calculated for the same conditions is shown in Fig.6.

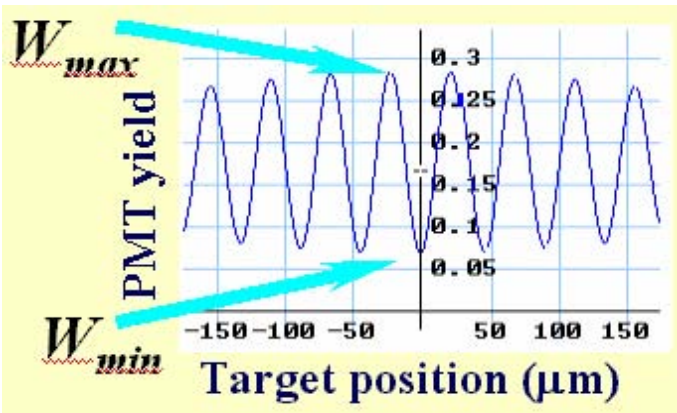

Figure 6: Calculated dependence of the radiation yield as a function of the vertical target position. 
In spite of the fact that this experiment was a test one, we can perform the vertical beam size estimation using the visibility:

$$
V=\frac{W_{\max }-W_{\min }}{W_{\max }+W_{\min }}
$$

As it was shown in the theory, the beam size may be found from equation:

$$
4 \pi \frac{\sigma \alpha}{\lambda}=\sqrt{\frac{\ln \left(V^{-1}\right)}{2}}
$$

and for the experimental result shown on Fig. 5 the beam size value is $\sigma=8.4 \pm 1 \mu m$

Here we neglect the contribution of second order factors (horizontal beam size, background and so on). A better spatial resolution and single shot measurement may be realized using intensified CCD camera.

\section{METHOD EXTENSION FOR SLAC FFTB}

Main feature of the SLAC FFTB electron beam is the large value of the Lorentz-factor $\gamma=60000$ in contrast to KEK ATF where $\gamma=2505$.

The first consequence of this feature is the extremely pre-wave zone for possible detector position. The far field zone criteria for a distance $L$ between target and detector is: $L>\gamma \lambda$. . For SLAC FFTB this condition results in the limitation $L>1 \mathrm{~km}$.

The following requirements due to the large value of Lorentz-factor must be fulfilled:

\section{Target Parameters}

Target polarization region $\gamma \lambda \approx 30 \mathrm{~mm}$ leads to requirement of target size $\approx 30 \mathrm{~mm}$. The beam size value being equal to $5 \mu \mathrm{m}$ requires an optimal angle between the target halves of $2 \alpha \approx 10 \mathrm{mrad}$. Requirement to the slit width $\mathrm{a}<\gamma \lambda$ results in $\mathrm{a}<6 \mathrm{~mm}$

\section{Optics}

A cylindrical lens is also necessary.

\section{Detector}

The total ODR yield does not depend on $\gamma$, therefore the intensified CCD camera may be used.

A bunch population range of 1010 may allow us to use this method for a single bunch beam size measurement.

\section{REFERENCES}

[1] P.Karataev, S.Araki, R.Hamatsu, et.al. Beam-Size Measurement with Optical Diffraction Radiation at KEK Accelerator Test Facility. PHYSICAL REVIEW LETTERS, 93, 244802 (2004).

[2] P.Karataev, S.Araki, R.Hamatsu, H.Hayano, T.Muto, G.Naumenko, A.Potylitsyn, N.Terunuma, J.Urakawa, Nucl. Instr. And Meth. B 227 (2005) 158.

[3] M.Castellano. Nucl. Inst. and Meth. A 394(1997) 275.

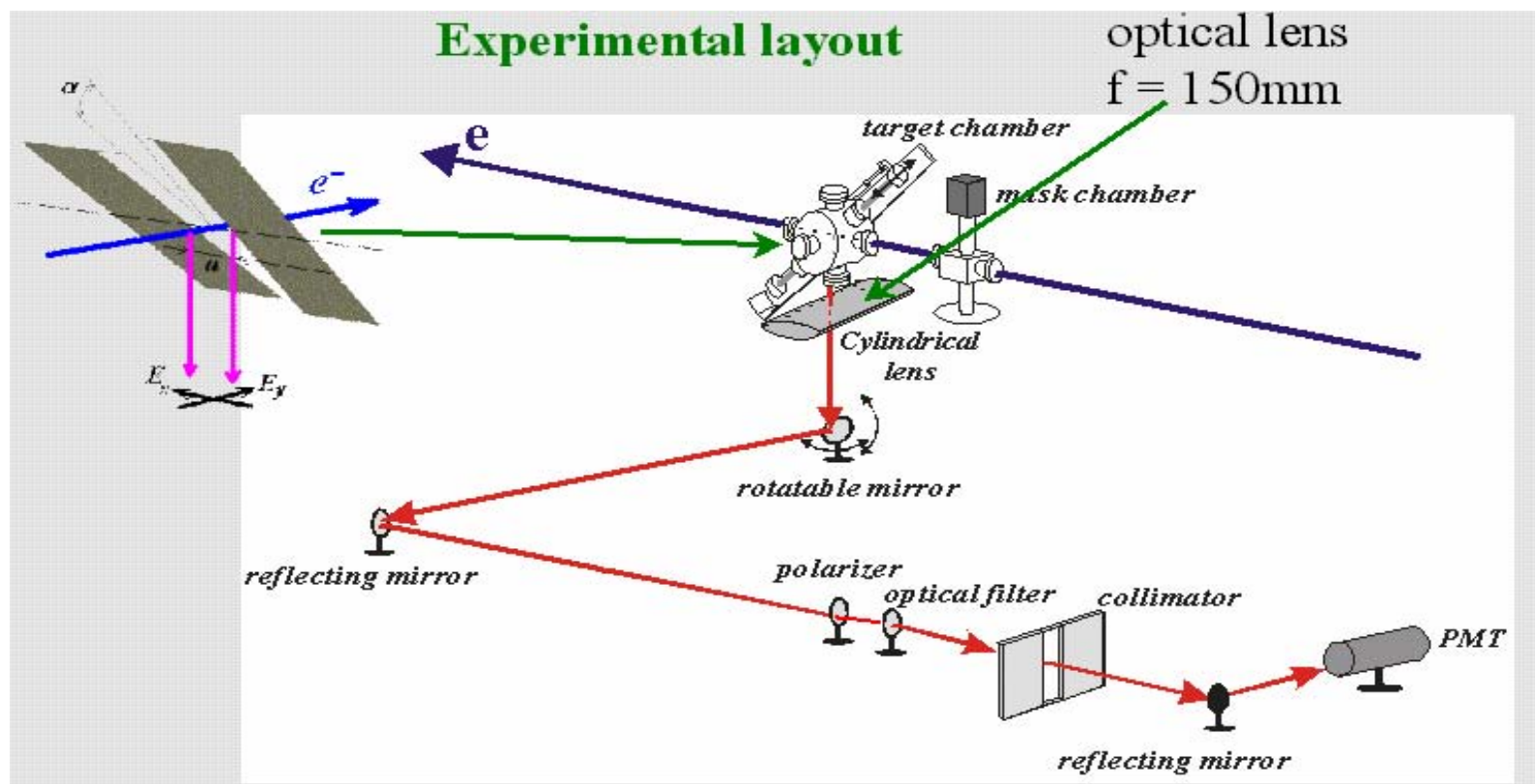

Figure 7: Principal scheme of experimental layout. 